\title{
PSA:154-163(155L) Peptide Vaccine
}

National Cancer Institute

\section{Source}

National Cancer Institute. PSA:154-163(155L) Peptide Vaccine. NCI Thesaurus. Code C29338.

A cancer vaccine comprised of a synthetic peptide with an amino acid sequence corresponding to positions 154-163 of the amino acid sequence for prostate-specific antigen (PSA) with a leucine substitution at position 155. Upon administration, PSA:154163 (155L) peptide vaccine may elicit a cytotoxic T-cell response against tumor cells that express PSA. ( $\mathrm{NCl05)}$ 